\title{
Laccase Gene Sh-lac Is Involved in the Growth and Melanin Biosynthesis of Scleromitrula shiraiana
}

\author{
Zhiyuan Lü, Xin Kang, Zhonghuai Xiang, and Ningjia He
}

State Key Laboratory of Silkworm Genome Biology, Southwest University, Chongqing 400716, China. Accepted for publication 27 October 2016.

\begin{abstract}
Scleromitrula shiraiana causes the popcorn disease in mulberry trees resulting in severe economic losses. Previous studies have shown that melanin may play a vital role in establishing the pathogenicity of fungi. In the present study, we identified the melanin produced in $S$. shiraiana belongs to DHN melanin by gas chromatography-mass spectrometry, and cloned the laccase Sh-lac, a potential DHN melanin biosynthesis gene from S. shiraiana. We obtained two stable Sh-lac silenced transformants using RNAi, ilac-4 and 8 to elucidate the DHN melanin biosynthetic

pathway in S. shiraiana. The melanin production of ilac-4 and ilac-8 was significantly reduced, and their vegetative growth was also suppressed. Results such as these led to a proposal that Sh-lac played a key role in DHN melanin formation in S. shiraiana and may function differentially with other melanin biosynthetic genes. The inhibition of melanin was accompanied by the decrease of oxalic acid and the adhesion of hyphae was impaired. Our results indicated that laccase was an important enzyme in the synthesis of melanin and might play a critical role in the pathogenicity of $S$. shiraiana.
\end{abstract}

Mulberry trees (Morus sp.) are cultivated widely in the temperate, subtropical, or tropical regions of the world, and can grow in a wide range of climatic, topographical, and soil conditions (Ercisli and Orhan 2007; Özgen et al. 2009). Mulberry trees have been grown to rear silkworms for a long time, but recently there has been a surge in the demand for mulberry fruits owing to their taste and nutritive value. Mulberry fruits also contain phytonutrient compounds like polyphenol pigment antioxidants, minerals, and vitamins that have proven health benefits. Mulberry alkaloids can activate macrophages. Macrophages then stimulate the human immune system to fight against health threats (Kim et al. 2013). However, mulberry fruits are susceptible to diseases from pathogens such as Ciboria carunculoides (Siegler and Jenkins) Whetzel, Ciboria shiraiana (Henn.) Whetzel, and Scleromitrula shiraiana (Henn.) Imai (Kishi 1998; Kohn and Nagasawa 1984; Whetzel and Wolf 1945). The fruit bodies of these pathogens release ascospores to infect blossoms and then cause the mummification of fruits (Fig. 1). This kind of disease is also called popcorn disease (Blain 1931; Siegler and Jenkins 1923). Traditional methods are not effective in the prevention and treatment of the disease.

The pathogen S. shiraiana, SX-001 strain, was isolated from diseased mulberry fruits. S. shiraiana produces large amounts of black polymer-like material on potato dextrose agar (PDA) medium and the sclerotia formed in the diseased fruit is coated with a thick black substance (Li and Rollins 2009). These black substances have the chemical properties of melanin, such as black color, insolubility in cold or boiling water and organic solvents, resistance to degradation by hot or cold concentrated acids, bleaching by oxidizing agents, and solubilization and degradation by hot alkali solutions (Butler and Day 1998; Wu et al. 2008). Butler et al. (2009) confirmed that the melanin produced by Sclerotinia sclerotiorum is a 1,8-dihydroxynaphthalene (1,8-DHN). Melanins are biological macromolecules formed by oxidative polymerization of phenolic

Corresponding author: N. He; E-mail address: hejia@swu.edu.cn

*The $\boldsymbol{e}$-Xtra logo stands for "electronic extra" and indicates that nine supplementary figures and two supplementary tables are published online.

(c) 2017 The American Phytopathological Society or indolic compounds that form complexes with proteins and carbohydrates (Langfelder et al. 2003; Watt et al. 2009). Melanin plays a protective role against various adverse external environmental conditions such as UV irradiation, osmotic pressure, and biotic stresses (Butler and Day 1998; Henson et al. 1999). Melanin is also closely related with pathogenicity of some plant and animal pathogens (Butler et al. 2001; Ebbole 2007; Jacobson 2000; Langfelder et al. 2003; Thomma 2003; Thywissen et al. 2011).

Fungi synthesize DHN (1,8-DHN) melanin and L-3,4dihydroxyphenylalanine (L-dopa) melanin. DHN melanin is synthesized by a polyketide pathway and is produced by a series of redox reactions (Gomez and Nosanchuk 2003). The scytalone dehydratase (SCD), 1,3,6,8-tetrahydroxynaphthalene reductase (4THNR), and/or 1,3,8-trihydroxynaphthalene reductase (3THNR) are important enzymes involved in redox reactions in DHN melanin biosynthesis pathway (Andersson et al. 1996; Lee et al. 2003). The final step of DHN polymerization to DHN melanin is putatively catalyzed by laccases (Tetsch et al. 2005; Upadhyay et al. 2013) (Fig. 2). In contrast, the L-dopa melanin is synthesized by laccases, and its production requires exogenously supplied phenolic precursors such as L-dopa (Williamson et al. 1998). However, the study of laccase catalyzed DHN polymerization reaction is rare. A mutant of putative laccase, Pgrl, failed to form melanin, which suggested Pgrl involved in the oxidation of DHN in Cochliobolus heterostrophus (Tanaka et al. 1991, 1992). One recent study demonstrated that a laccase (LAC2) was associated with melanin production and pathogenicity in Colletotrichum orbiculare (Lin et al. 2012). Laccase is a coppercontaining enzyme that catalyzes the oxidation of a phenolic substrate by coupling it to the reduction of oxygen to water (Liu and Nizet 2009). Laccases are ubiquitously found in many organisms and are involved in many other biological activities (besides melanin biosynthesis) such as in morphogenesis, pathogenesis, and lignin degradation (Baldrian 2006). For example, a laccase-like enzyme, relative to the fungal pathogenicity in Botrytis cinerea, can degrade several phytoalexins of grapevines (Sbaghi et al. 1996). In the present study, we isolated S. shiraiana, one of the pathogens causing the mulberry popcorn diseases to study its morphology and biochemistry. We hypothesize that the copious amounts of melanin produced by the fungus are related to the pathogenicity of the fungus. We investigated the roles of a laccase gene Sh-lac in the 
melanin biosynthetic pathway of S. shiraiana. Our results will help understand the etiology of the popcorn disease at the molecular level. Development of an agent that can specifically inhibit the production of melanin or degradation of melanin will be an effective means for prevention and control of the mulberry popcorn disease.

\section{MATERIALS AND METHODS}

Isolation of fungal strains and culture conditions. The diseased mulberry fruits were collected from the mulberry orchard in Southwest University, Chongqing, China. The drupelets of diseased mulberry fruit were rinsed with water, air-dried, and disinfected with $70 \%$ alcohol for $30 \mathrm{~s}$ followed by $0.1 \%$ mercuric chloride surface-sterilized for 5 min (Zhang et al. 2009). The drupelets were then rinsed five times with sterile distilled water to ensure complete cleansing of alcohol and mercuric chloride. A sterile scalpel was used to slice the drupelets, which were then placed on the PDA medium and incubated at $25^{\circ} \mathrm{C}$ in darkness.

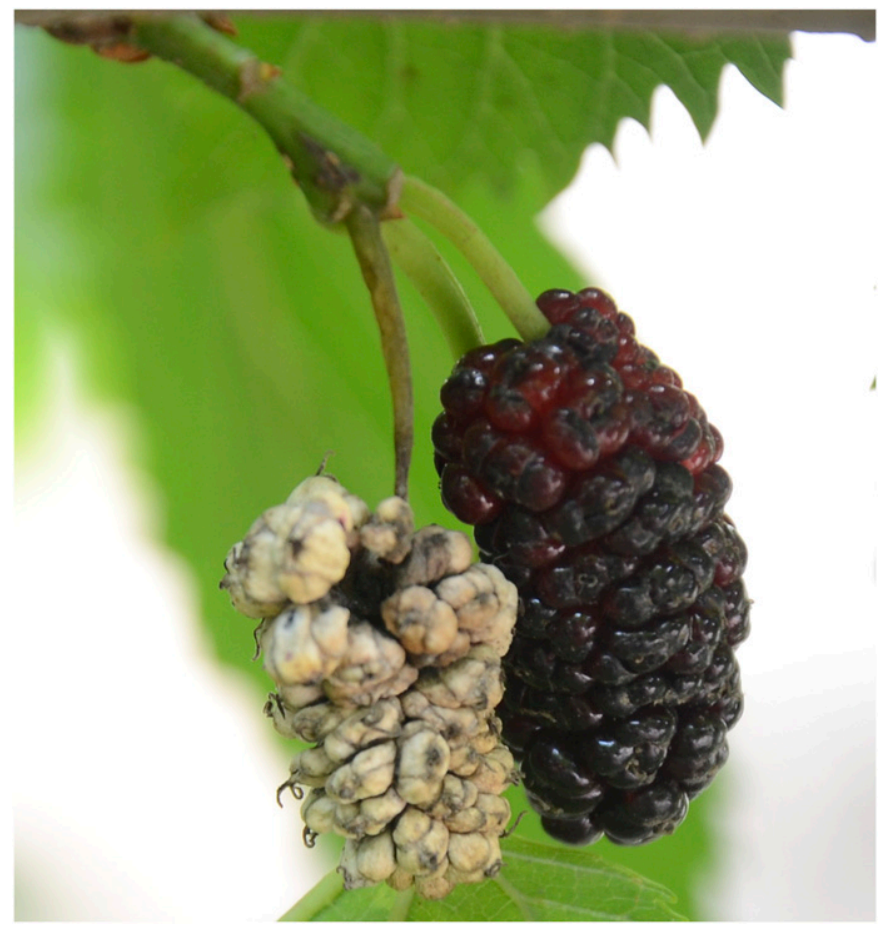

Fig. 1. The symptom of mulberry popcorn disease. The white mummified mulberry fruit.
The target pathogen was isolated and named SX-001 strain. The strain was routinely subcultured on PDA at $25^{\circ} \mathrm{C}$ to maintain vigor and purity. A sterile cellophane membrane was placed on the medium before incubation to collect the hyphae from cultures grown on solid medium.

Nucleic acid isolation, cDNA production, and real-time RT-PCR analysis. The hyphae were collected, frozen in liquid nitrogen, and stored at $-80^{\circ} \mathrm{C}$ until further use. Genomic DNA was isolated according to a previously described method of Yelton et al. (1984). Total RNA was extracted with the RNAiso Plus (Takara, Dalian, China) according to the manufacturer's instructions and treated with RNase-free DNase I to remove contaminated DNA. First-strand cDNA was synthesized using a PrimeScript RTase based PrimeScript RT-PCR Kit (Takara, Dalian, China). For RT-PCR, $1 \mu \mathrm{g}$ of total RNA was reverse transcribed as the template. Real-time RT-PCR was performed using SYBR Green (Takara) on StepOnePlus Real-Time PCR system (Applied Biosystems, Foster City, CA). The expression level of $S h$-lac was analyzed via qPCR with the primer pairs RT-LAC02F/RT-LAC02R. The cDNA abundance in the sample was normalized using the actin housekeeping gene as a control. S. sclerotiorum actin gene (SS1G_08733) primers pair ActinF/ ActinR were used to amplify the actin gene from $S$. shiraiana cDNA (Yu et al. 2012). PCR amplification and sequencing showed that this pair primer is suitable for amplified actin gene in S. shiraiana.

Melanin preparation. S. shiraiana inocula were maintained on PDA and then inoculated into a $250-\mathrm{ml}$ flask containing $100 \mathrm{ml}$ of malt extract (ME) medium and incubated at $25^{\circ} \mathrm{C}$ for 17 days with continuous shaking at $200 \mathrm{rpm}$. Melanin was prepared and purified as described by Wu et al. (2008), with slight modification as chloroform and isoamyl alcohol (1:5) were replaced with chloroform and ethyl alcohol (1:1).

Sample preparation and melanin identification. Inquiry sample and standard were dissolved in acetonitrile and then centrifuged at 10,000 rpm for $10 \mathrm{~min}$. Supernatant $(100 \mu \mathrm{l})$ was rotary evaporated to dryness. Derivatization was performed with $50 \mu \mathrm{l}$ of methyl-trimethyl-silyl-trifluoroacetamide at $37^{\circ} \mathrm{C}$ for $1 \mathrm{~h}$. Samples were analyzed by gas chromatography-mass spectrometry (GC-MS) (Agilent Technologies Inc., CA). The separation was done with a DB-5MS column $(30 \mathrm{~m} \times 0.25 \mathrm{~mm}$ I.D. with a $0.25 \mu \mathrm{m})$. The injection volume was $1.0 \mu \mathrm{l}$ and carrier gas was helium at $1.0 \mathrm{ml} / \mathrm{min}$. The oven program started at an initial temperature of $50^{\circ} \mathrm{C}$ for $3 \mathrm{~min}$. Temperature was then increased at a rate of $5^{\circ} \mathrm{C} / \mathrm{min}$ up to $300^{\circ} \mathrm{C}$, maintained at $300^{\circ} \mathrm{C}$ hold for 3 min with a $10: 1$ split ratio. Ionization was performed by electronic impact (EI), setting the electron multiplier to $70 \mathrm{eV}$.

Construction of gene silencing vectors and $S$. shiraiana transformation. The pCIT vectors were kindly provided by Yang $\mathrm{Yu}$ (College of Plant Protection, Southwest University, Chongqing,

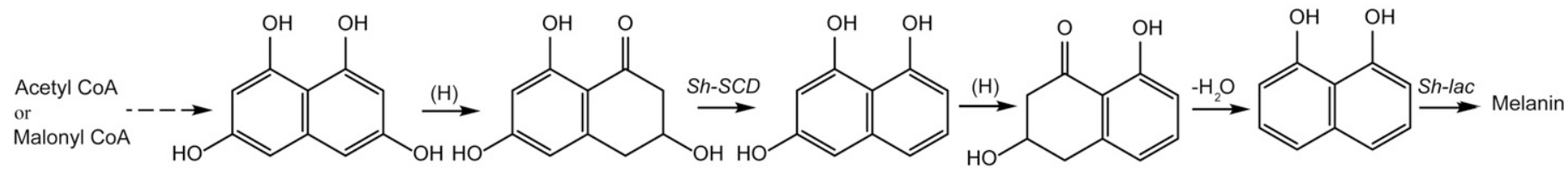<smiles>O=C1C(=O)c2cc(O)cc(O)c2C(=O)C=C1O</smiles>

Fig. 2. 1,8-Dihydroxynaphthalene melanin biosynthetic pathway of Scleromitrula shiraiana. 
China). The primer pair idz LAC-F and idz LAC-R were used to amplify a 417-bp Sh-lac coding sequence to obtain the sense fragments to construct the Sh-lac silencing vector. We introduced two restriction sites HindIII and BamHI at the $5^{\prime}$ terminus of the sense primer, and another two restriction sites EcoRI and PstI at the 5 ' terminus of the antisense primer. The primer sequences are listed in Supplementary Table S1. The PCR product was sequenced and digested with PstI and BamHI, and then ligated into vector pCIT (Yu et al. 2012) between the intron and $\mathrm{T} t r p C$, to produce the intermediate vector pCIT idzLAC-I. The same PCR products were digested with HindIII and EcoRI and then ligated into previous constructed intermediate vector pCIT idzLAC-I between the intron and PtrpC to yield vector pCIT idzLAC-II. The pCIT idzLAC-II was digested with $\mathrm{XhoI}$ and $\mathrm{SacI}$ to obtain a fragment in which the two 417-bp fragments were inserted in opposite orientations between PtrpC and TtrpC. This fragment was then ligated between $X h o \mathrm{I}$ and SacI sites of pCAMBIA3300, which carries hygromycin B phosphotransferase gene, to create the Sh-lac silencing vector pCHidzLAC. The vector pCHidzLAC was transformed into the wild-type strain $\mathrm{SX}-001$. The candidate transformants were screened on PDA containing hygromycin B at $30 \mu \mathrm{g} / \mathrm{ml}$. The vector $\mathrm{pCHidzSCD}$ was constructed for silencing $S h-S C D$ using the same method.

Melanin production assay. $S$. shiraiana mycelia were preserved on PDA. Each flask, containing $50 \mathrm{ml}$ of ME medium, was inoculated with five equal-sized mycelium (to reduce systematic errors caused by the operation) and incubated at $25^{\circ} \mathrm{C}, 200 \mathrm{rpm}$ for 17 days. Three replicates were performed per treatment. ME medium was filtered through two layers of filter paper (Sangon Biotech, Shanghai, China). The filtrate was scanned using a DU800 spectrophotometer (Beckman Coulter). Melanin sample extracted from the mycelium showed that absorbance at wavelengths from 200 to $400 \mathrm{~nm}$ was interfered by the liquid medium. Also absorbance was low at wavelengths greater than $500 \mathrm{~nm}$, and this could affect the accuracy of the detection results. Thus, $420 \mathrm{~nm}$ was selected to measure the yield of melanin.

Chromatography. Chromatographic separation of oxalic acid was performed using Waters Acquity UPLC I-CLASS system (Waters Corp., MS); ACQUITY UPLC HSS T3 column $(1.8 \mu \mathrm{m}$, $2.1 \times 50 \mathrm{~mm})$ was used. The mobile phase used was: KH2PO4 $(90 \%$ $\mathrm{A} ; 0.5 \% \mathrm{wt} / \mathrm{vol}, \mathrm{pH} 2.0)$ and TBA (10\% B; tetrabutylammonium, $20 \mathrm{mM}$, do not adjust $\mathrm{pH}$ ) at a flow rate of $0.5 \mathrm{ml} / \mathrm{min}$. The chromatogram was monitored at a wavelength of $210 \mathrm{~nm}$. The column temperature was maintained at $30^{\circ} \mathrm{C}$ and the injection volume of each sample and standard solution was $0.8 \mu \mathrm{l}$. Data were processed with Waters Empower 2 software for analysis.

\section{RESULTS}

Isolation and identification of $\boldsymbol{S}$. shiraiana. We identified $S$. shiraiana from the diseased mulberry fruit through internal transcribed spacer (ITS) alignment using the primer pair ITS1/ITS4 (White et al. 1990). The ITS sequences cloned from the genome of $S$. shiraiana's apothecia confirmed its identity (Supplementary Fig. $\mathrm{S} 1)$. We named it SX-001 strain. The $S$. shiraiana colony darken when cultured for about 4 days on PDA medium. The colony almost stopped growing on the tenth day and showed remarkable blackening (Fig. 3A and B). At 20 to 25 days, we saw the appearance of milky liquids on the colony surface (Fig. 3C and D). The milky liquids contain many microspores, which germinated to form colonies. Sclerotia typically have two structures: the hard black outer layer is called the pseudoparenchyma and the loose inner layer is called the prosenchyma (Fig. 2F to I). Sclerotia are the dormant pathogen structures and only overwintered sclerotia have the vitality to form fruit bodies.

$S$. shiraiana hyphae disorganize mulberry drupelets and form sclerotia in diseased fruits. S. shiraiana ascospores are the only source of primary infection and infect mulberry inflorescences. The disease manifested itself with the growing mulberry fruits, and we observed the formation of sclerotia in drupelet. The mulberry fruits finally turned to mummified white fruits. We observed fibrous, interwoven, and interspersed hyphae in the paraffin sections of diseased mulberry fruits, and the hyphae disorganize drupelet tissue into small granules (Fig. 4A). The small granules in the drupelet tissue provide adequate nutrition for $S$. shiraiana.

Paraffin sections produced at different stages of the disease showed a large number of small black particles gathered around a black spheroid structure (Fig. 4B). The small black particles are ingredients of the outer wall of sclerotia. These black particles probably shed from the mycelium (Fig. 4C) and are parts of the fractured hyphae.

Identification of melanin. Extract ion chromatography (EIC) studies identified the black substance from $S$. shiraiana hyphae as $1,8-\mathrm{DHN}$, which is a monomer of DHN melanin. The results
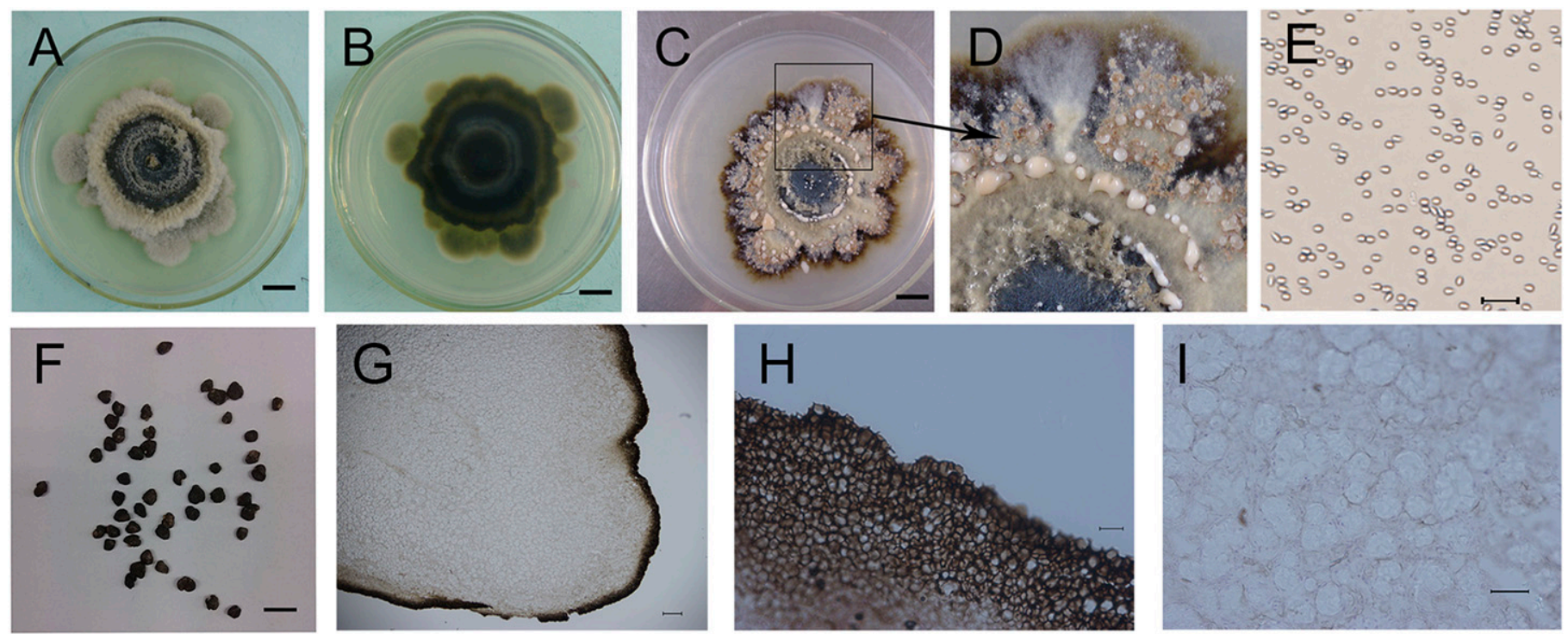

Fig. 3. Morphology of Scleromitrula shiraiana. A and B, The front and reverse of $S$. shiraiana grown on potato dextrose agar (PDA) medium for 10 days and colony was blackened. Bar: $1 \mathrm{~cm}$. C, S. shiraiana grown on PDA and much liquid secreted containing microspores after 25 days. Bar: $1 \mathrm{~cm}$. D, A partial enlarged view of the upper end of the colonies in C. $\mathbf{E}$, Microspore micrographs are in the optical microscope. Bar: $20 \mu \mathrm{m}$. F, The sclerotia of pathogens of mulberry popcorn disease. Bar: $1 \mathrm{~cm}$. G, Overall microstructure sclerotia. Bar: $200 \mu \mathrm{m}$. H, Sclerotia pseudoparenchyma and black particles. Bar: $50 \mu \mathrm{m}$. I, Sclerotia prosenchyma. Bar: $40 \mu \mathrm{m}$. 
showed that DHN appeared at 24.4 min as shown on the EIC chart (Fig. 5A). DHN can be detected at the same site from inquiry melanin sample. Mass spectrometric analysis indicated $S$. shiraiana melanin was indeed DHN melanin (Fig. 5). In addition, S. shiraiana was treated with tricyclazole (5-methyl-1,2,4-triazol[3,4]benzothiazole). Tricyclazole is an agrochemical that acts specifically on DHN melanin pathway by inhibition reductase, 1,3,6,8-tetrahydroxynaphthalene reductase (4THNR) and/or 1,3,8-trihydroxynaphthalene reductase (3THNR). The results showed that tricyclazole at $60 \mu \mathrm{g} / \mathrm{ml}$ significantly inhibited the growth of fungi and the melanin production of S. shiraiana (Supplementary Fig. S2).

Cloning of the Sh-lac from $S$. shiraiana. We cloned the laccase gene to identify the laccase responsible for melanin biosynthesis and pathogenicity of $S$. shiraiana. We obtained an approximately 1,240-bp amplified fragment from $S$. shiraiana cDNA using a pair of degenerate primers targeted to conserved regions of the fungal laccase gene. Sequence BLAST analysis of the fragment exhibited $97 \%$ identities to the laccase (SS1G_11927) of S. sclerotiorum 1980 ,
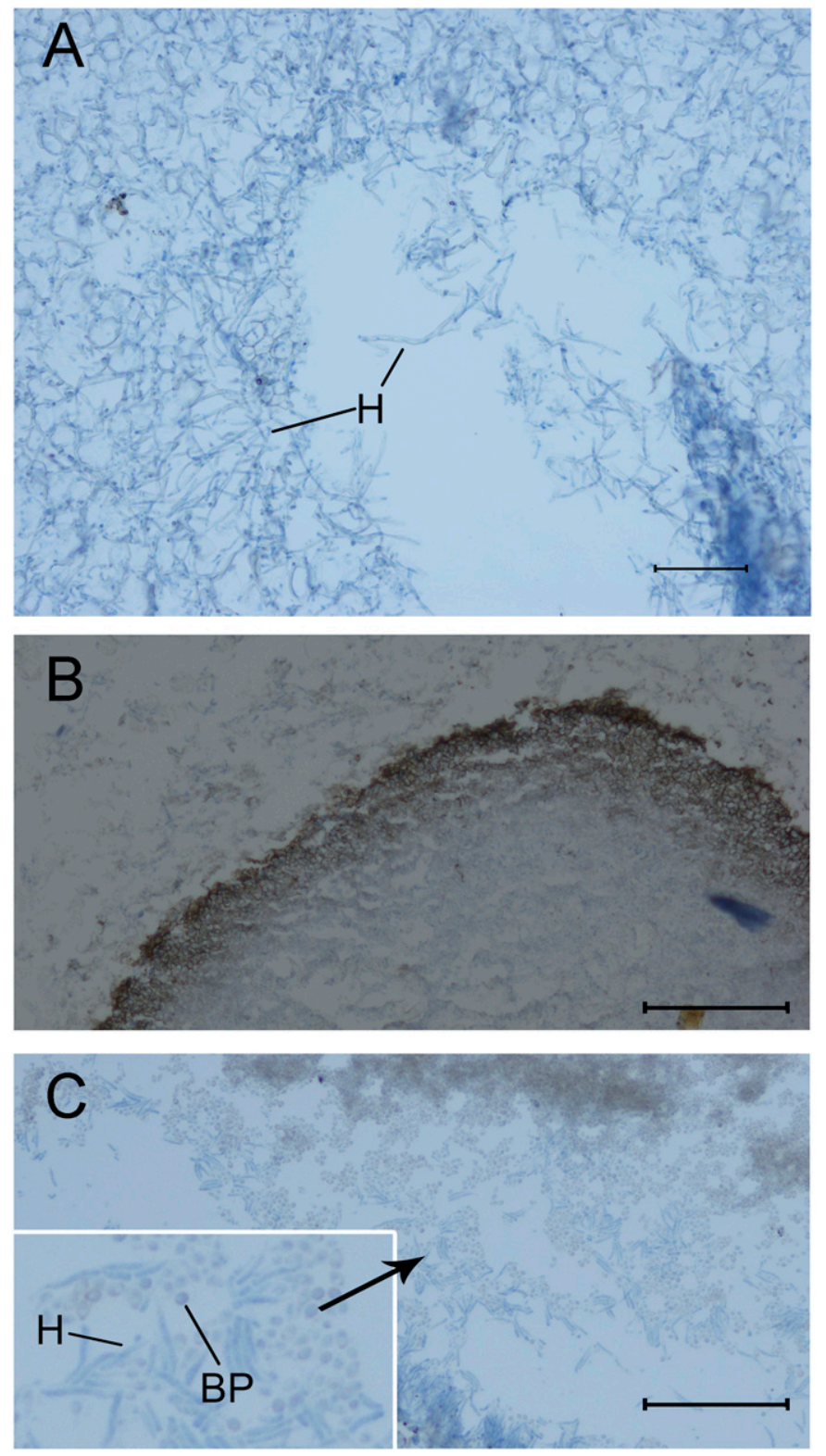

Fig. 4. Mulberry drupelet paraffin sections. A, Infected drupelet that was full of hyphae. B, Sclerotia is forming in diseased fruit and sclerotia pseudoparenchyma is composed of small black particles. $\mathbf{C}$, Small black particles are formed by the fragmentation from fracture hyphae. Bar: $100 \mu \mathrm{m}$. H: hyphae, BP: black particle. indicating that it encoded a homolog of laccase. We named this homolog Sh-lac. The full-length Sh-lac gene contains an open reading frame (ORF) of 2,016 bp, and encodes 672 amino acids. The Sh-lac ORF comparison with the laccase gene sequence amplified from genomic and cDNA revealed one intron (86 bp) with the consensus GT-AG rules (Breathnach et al. 1978; Mount 1982). A putative signal peptide consisting of 18 amino acid residues was identified at the $\mathrm{N}$ terminus (Fig. 6A). It predicted that Sh-lac protein is an extracellular protein. We found three typical cupredoxin domains in laccase: Cu-oxidase_3 (PF07732), Cu-oxidase (PF00394), and Cu-oxidase_2 (PF07731) (Fig. 6A). The degree of overall sequence identity of putative Sh-lac and putative laccase protein BC1G_10329 of Botrytis cinerea $\mathrm{B} 05.10$, hypothetical protein SS1G_11927 of S. sclerotiorum 1980 was approximately 72 and $77 \%$, respectively. But, Sh-lac protein showed high sequence conservation in sequence alignment with Magnaporthe oryzae (MGG_13464) and S. sclerotiorum laccases (Supplementary Fig. S3). Our study provides sufficient evidence that Sh-lac is a laccase of S. shiraiana. The neighbor-joining phylogenetic analysis of Sh-lac sequence with other plant-pathogenic fungi laccase sequences showed that all laccases were clustered in two groups. Ascomycetes are in group I, while basidiomycetes are in group II (Fig. 6B).

High expression level of Sh-lac is related to melanin production in S. shiraiana. RT-PCR studies showed that the expression level of Sh-lac increased from the fourth day forward until the twelfth day (Supplementary Fig. S4). This increase coincided with the beginning of fungal colony blackening or production of melanin. On the second day, when the mycelia were completely white, the Sh-lac expression level was low. The expression of Sh-lac started to increase from the fourth day, when the colony began to darken and continued to increase until the twelfth day, when the fungal colony stopped growing, and the melanin production was reduced.

Sh-lac silenced transformants show decrease in melanin production. We transformed the $S$. shiraiana wild-type strain SX001 using the vector pCHidzLAC (Fig. 7A), wherein the Sh-lac gene was transformed. Segmental amplification of hygromycin B phosphotransferase gene (Fig. 7B) confirmed eight transformants. We named the transformants ilac-1 to ilac-8. Sh-lac silencing caused the formation of abnormal colonies on PDA (Fig. 7C and D). Transformant colonies changed color from dark olive brown (WT) to maroon or light brown. We hypothesized that a lighter color of colonies was associated with a lower production of melanin. In addition, transformant hyphae were fluffy. We examined the relative expression levels of Sh-lac in the transformants and WT strain SX-001 after multiple subculture (Fig. 7E).

We chose stable transformants ilac- 4 and ilac- 8 for further studies (Fig. 8B). The melanin productions in these two transformants were significantly lower than WT and Pint (empty vector transformant) by measuring the filtrate absorbance at $420 \mathrm{~nm}$ (Fig. 8A and C, Supplementary Fig. S5). Also notable was that the colonies adhesion of ilac-4 and ilac- 8 was reduced on the PDA. Although it is difficult to measure the adhesion of colonies on PDA, it is widespread and the colonies rolled up or separated from the PDA medium in ilac-4 (9 of 10 colonies observed) and ilac-8 (10 of 10 colonies observed) (Supplementary Fig. S6). However, all of the WT mycelia were firmly rooted in the culture medium and were difficult to peel. The results indicated ilac- 4 and ilac- 8 attenuate adhesion compared with WT.

Silenced Sh-lac inhibited the vegetative growth of $S$. shiraiana. Vegetative growth was inhibited in the Sh-lac silenced transformants (Fig. 7C and D). In order to verify whether the vegetative growth and melanin are relevant in S. shiraiana, we obtained scytalone dehydratase (SCD) silencing transformant using the same method. $S h-S C D$ is another key gene in the DHN melanin biosynthesis pathway in $S$. shiraiana. The expression of $S h-S C D$ in resultant transformant iSCD-2 was $35.3 \%$ decreased compared with the WT. Meanwhile, the melanin production of iSCD-2 was also reduced. However, transformant iSCD-2 was a reddish brown colony on PDA 

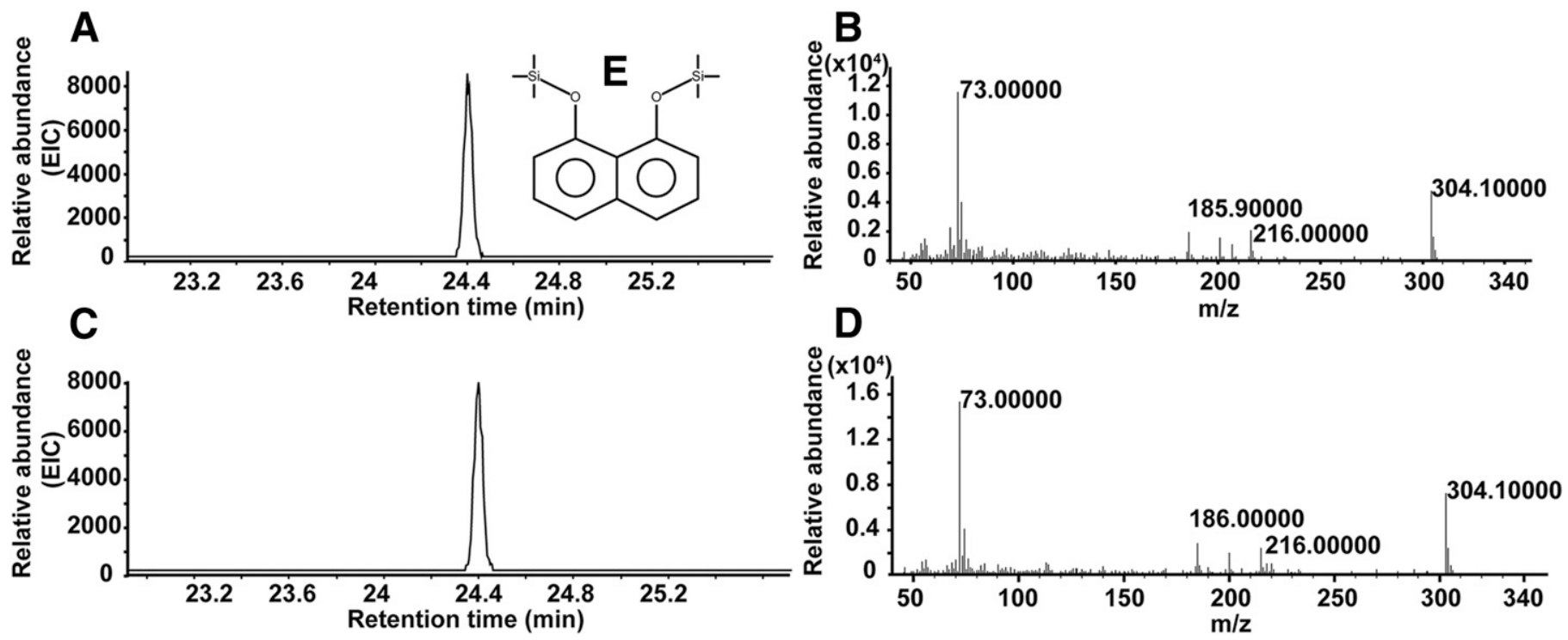

Fig. 5. Identification of inquiry melanin sample. A, Standard DHN extract ion chromatograpy (EIC) chart. B, Standard DHN mass spectrometry chart. C, Inquiry sample EIC chart. D, Inquiry sample mass spectrometry chart. E, DHN standard or inquiry melanin sample monomer derivatization.

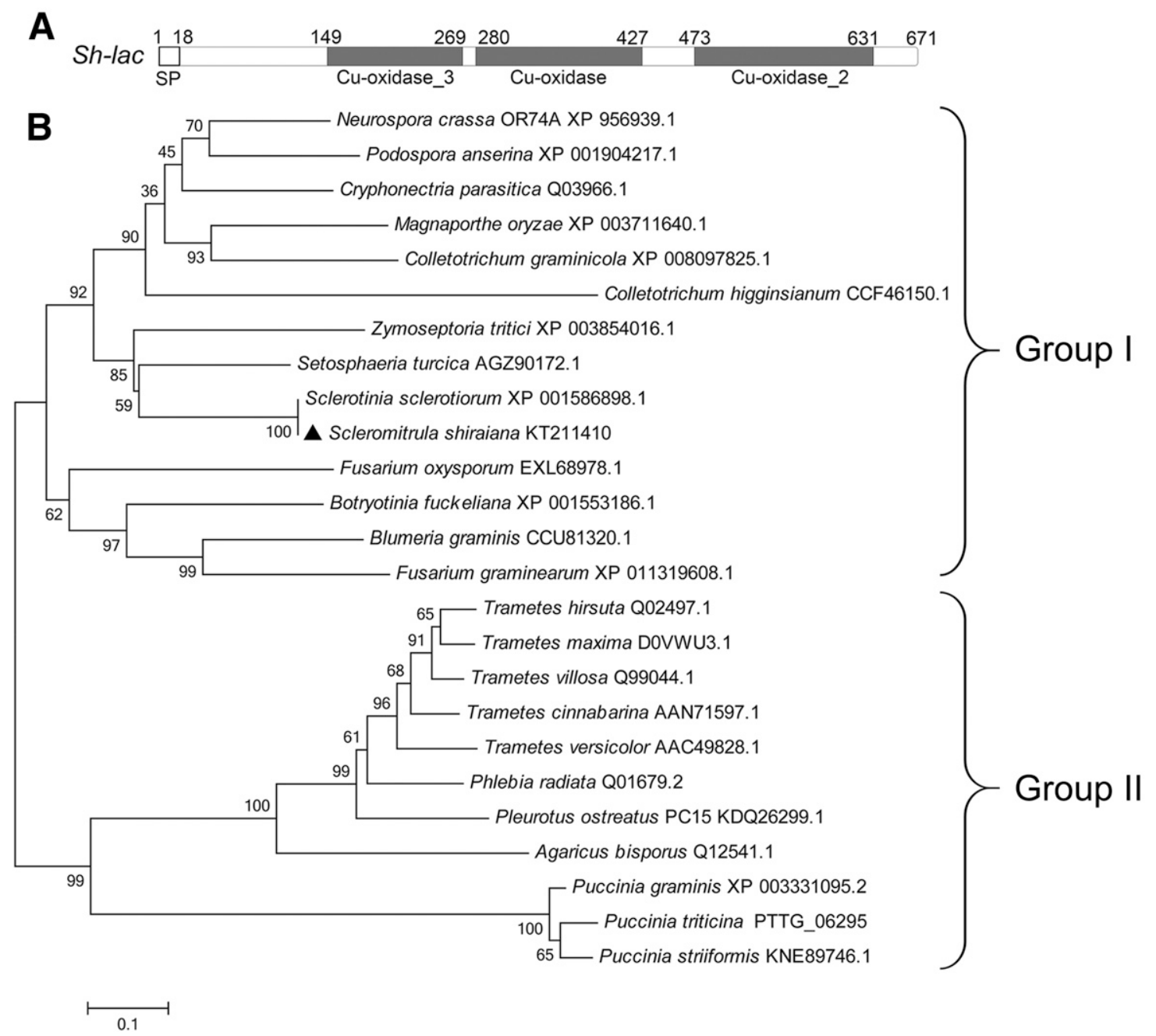

Fig. 6. Analysis of Sh-lac. A, Schematic representation of domains in Sh-lac protein. SP: signal peptide at the N-terminal. Sh-lac protein contains three multicopper oxidase domains: Cu-oxidase_3, Cu-oxidase, and Cu-oxidase_2. B, Neighbor-joining phylogenetic tree of Sh-lac and laccases from other fungi. The bootstrap values $(\%)$ obtained with 1,000 replicates are indicated on branches. Solid triangle indicates Sh-lac from Scleromitrula shiraiana. Fungi belong to ascomycetes in the group I, and belong to basidiomycetes in the group II. 
with normal vegetative growth (Supplementary Fig. S7). This indicated that the inhibition of vegetative growth was not related to the reduction of melanin in $S$. shiraiana.

Low melanin levels affect the virulence of $S$. shiraiana. It's a technical difficulty to test pathogenicity on mulberry fruit. Therefore, we indirectly tested transformant pathogenicity by detecting the oxalic acid that is a virulence factor in many sclerotia forming phytopathogens. We detected oxalic acid in culture filtrates of the different strains by ultra performance liquid chromatography (UPLC). WT strain contain $2.13 \mathrm{mM}$ oxalic acid, whereas CK (sterile ME medium), ilac-4, and ilac-8 did not produce measurable amounts of oxalic acid (Fig. 9; Supplementary Fig. S8). Meanwhile, the oxalic acid concentration of WT treated with tricyclazole $(0.49 \mathrm{mM})$ and iSCD-2 $(0.98 \mathrm{mM})$ was also significantly lower than that in the WT strain. Since oxalic acid production is proportional to melanin production, either directly or indirectly, our results suggest that melanin may very possibly be implicated in $S$. shiraiana pathogenicity.

\section{DISCUSSION}

Characteristics of $\boldsymbol{S}$. shiraiana. In the present study, we isolated strains of $S$. sharaiana that cause the popcorn disease in mulberry fruits. The strain was named SX-001strain and elucidated the mechanism of the formation of sclerotia in S. shiraiana-infected mulberry fruits. Paraffin sections of diseased fruits showed that the fungal hyphae invade the infected mulberry drupelet and destroy the host structure. After that, the hyphae disintegrate into small black particles, which make up the outer wall of the sclerotia. The sclerotia of pathogenic fungi are dormant structures with a hard, dense black wall that can withstand adverse environmental impacts (Amselem et al. 2011). Melanin has been implicated in sclerotia formation and pathogenicity in S. sclerotiorum (Butler et al. 2009), which is closely related to $S$. shiraiana.

S. shiraiana produces DHN melanin. Our GC-MS studies confirmed that $S$. shiraiana produces DHN melanin. The biosynthesis of DHN melanin starts with the synthesis of pentaketide and proceeds to 1,3,6,8-tetrahydroxynaphthalene (4THN) (Wheeler and Stipanovic 1979). Subsequently, tetrahydroxynaphthalene reductase (THNR) catalyzes the reduction of $4 \mathrm{THN}$ to generate scytalone. The scytalone dehydratase (SCD) then catalyzes the dehydration of scytalone to produce 1,3,8-trihydroxynaphthalene (3THN), the reduction of 3THN to vermelone, and the dehydration of vermelone to 1,8-DHN (Kihara et al. 2004a, b; Thompson et al. 2000; Vidal-Cros et al. 1994). Tricyclazole specifically inhibits the reductases involved in DHN melanin biosynthesis in black fungi (Andersson et al. 1996; Liao et al. 2001). The conidial pigmentation

A

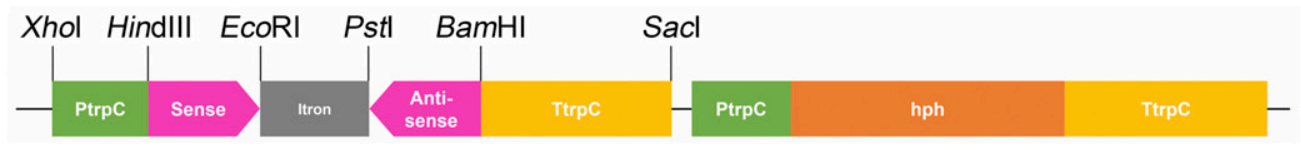

B

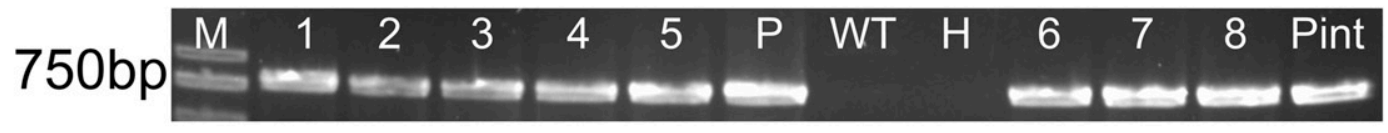

C
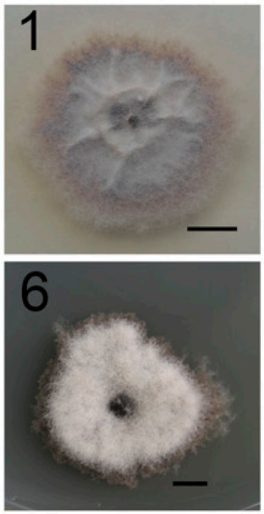

D

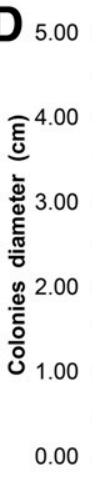
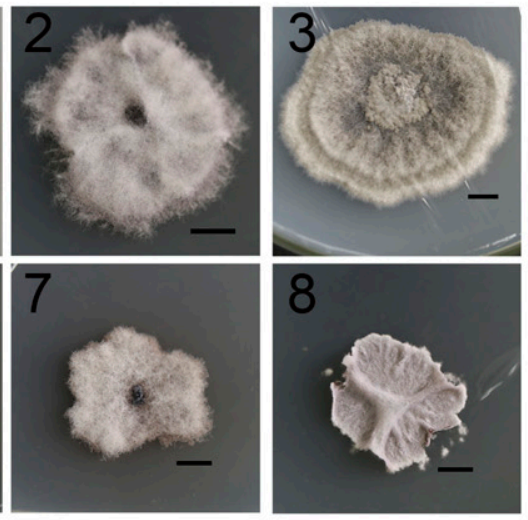

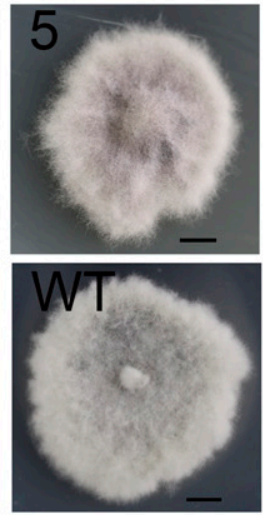

E

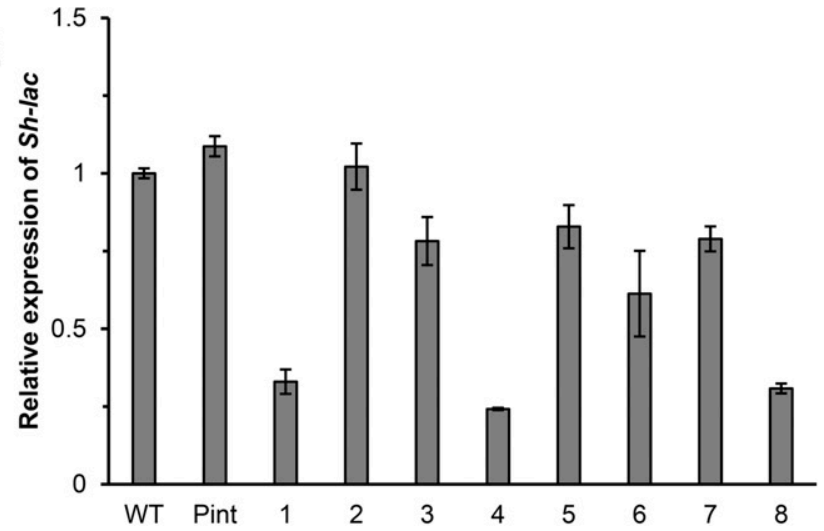

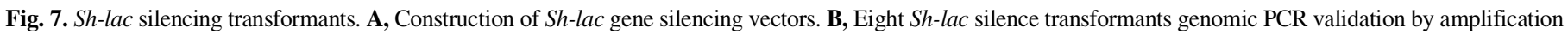

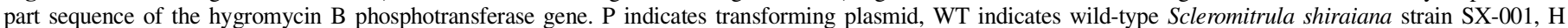

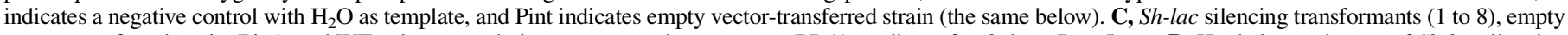

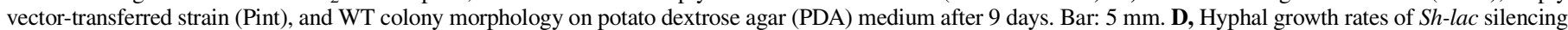

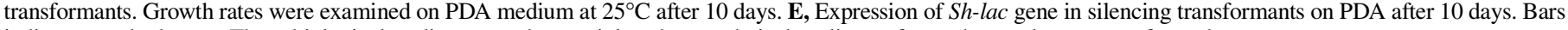
indicate standard error. Three biological replicates, each containing three technical replicates for each sample, were performed. 
of Colletotrichum lagenarium and $M$. oryzae are synthesized via DHN melanin pathway, and the pigmentations were restrained by tricyclazole treatment (Howard and Valent 1996; Perpetua et al. 1996). In the present study, tricyclazole at $60 \mu \mathrm{g} / \mathrm{ml}$ can significantly inhibit the growth and melanin production of $S$. shiraiana.

Sh-lac is not only involved in the synthesis of melanin, but also related to the vegetative growth. The $1,8-\mathrm{DHN}$ is oxidized and polymerized to form melanin (Bell and Wheeler 1986). We identified a laccase gene, Sh-lac-implicated in melanin synthesis-in S. shiraiana. Laccase is involved in the DHN polymerization reaction to form melanin in the DHN-melanin biosynthetic pathway (Lin et al. 2012; Upadhyay et al. 2013). Sh-lac has a predicted signal peptide and is an extracellular protein. Generally, melanin is located in either external or within the cell walls in fungi
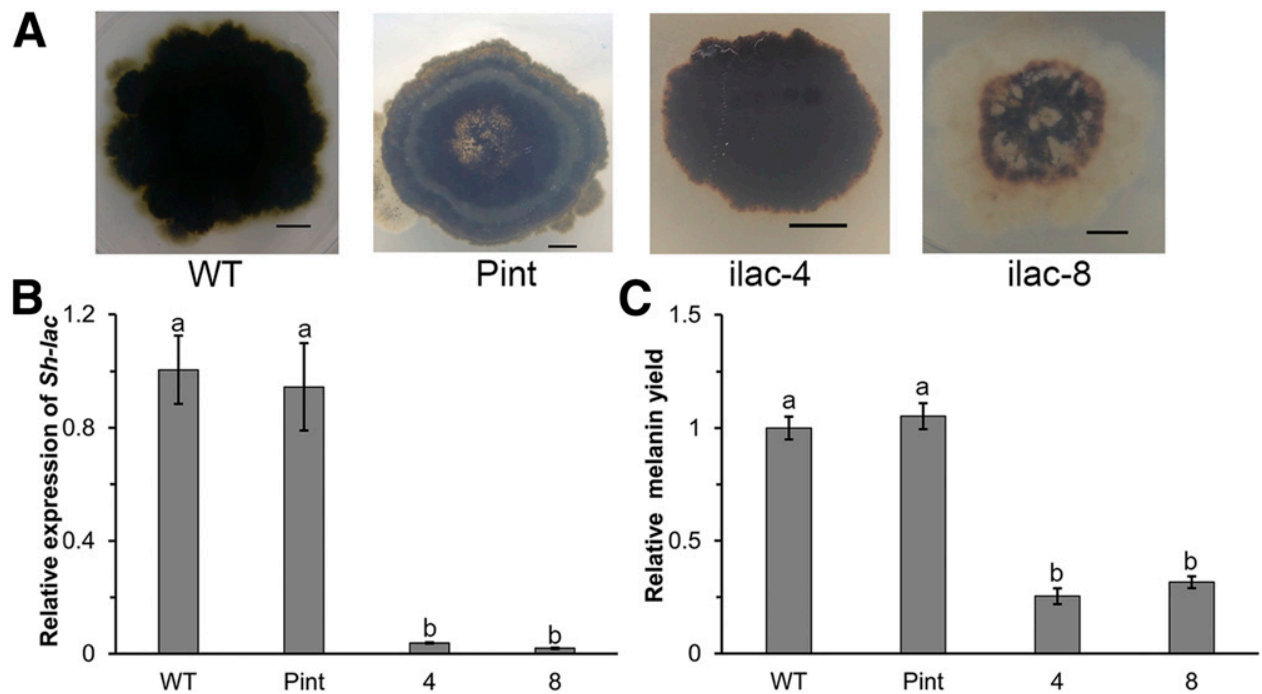

Fig. 8. Reduction of melanin in Sh-lac silencing transformants ilac-4 and ilac- 8 . A, WT, Pint (empty vector transformant), ilac-4, and ilac- 8 were incubated on potato dextrose agar for 17 days. The reduced melanin of colonies in silencing transformants is shown. Bar: $5 \mathrm{~mm}$. B, The relative expression levels of Sh-lac gene in transformants ilac- 4 and ilac- 8 . C, The relative melanin yield in transformants ilac- 4 and ilac-8. Letters a and $\mathrm{b}$ indicate statistical differences, $P<0.05$. The experiments were repeated at least three times.

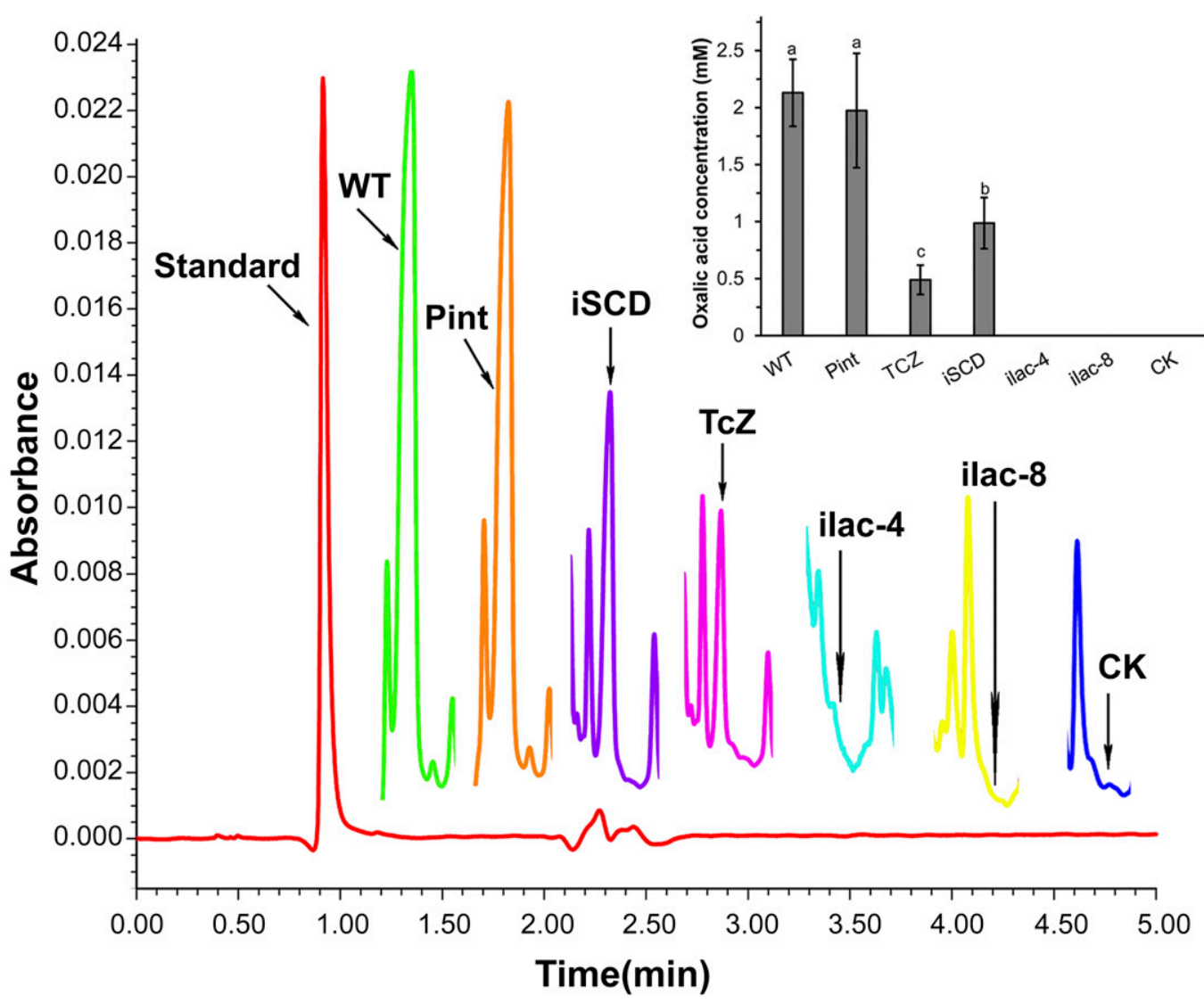

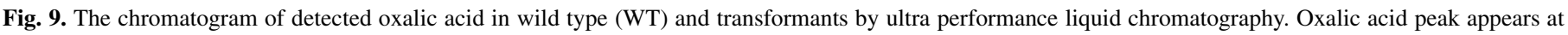

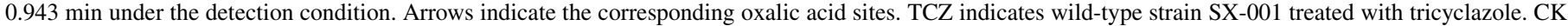
is a negative control (sterile malt extract medium). The experiments were repeated twice. 
(Ellis and Griffiths 1974). In order to verify the function of Sh-lac in the melanin synthesis, we silenced $S h$-lac gene by RNA interference (RNAi). Most of the Sh-lac silenced transformants had reduced melanin and slow growth rate. We selected two stable silenced transformants ilac- 4 and ilac- 8 for further study. The melanin yield of ilac- 4 and ilac- 8 and growth rate were significantly lower than WT. In order to clarify whether inhibition of the vegetative growth of $S$. shiraiana was associated with reduced melanin production by silencing $S h$-lac gene, another key gene $S h-S C D$ in DHN melanin biosynthesis pathway was silenced. The colony of iSCD-2 transformant was reddish brown with normal vegetative growth. The distinctive growth pattern of the transformant suggested the possibility that Sh-lac gene is involved in the vegetative growth of $S$. shiraiana. Laccase is a multifunctional factor in organisms. In addition to its participation in DHN melanin synthesis, laccase is also involved in morphogenesis, pathogenesis, and lignin degradation (Baldrian 2006). Transformants ilac-4 and ilac-8 are probably deficient in the morphogenesis, and lead to slow growth.

The fungus Gaeumannomyces graminis var. tritici is a phytopathogenic ascomycete that was known to produce DHN melanin and complete its infection with melanized hyphae (Edens et al. 1999). It has been reported that a purified laccase of G. graminis catalyzed the polymerization of $1,8-\mathrm{DHN}$ into melanin in vitro (Edens et al. 1999). In the present study, different strain filtrates and standard 1,8-DHN were used in assay. Compared with WT and Pint, the catalytic abilities of ilac-4 and ilac- 8 filtrate were decreased (Supplementary Fig. S9). These results also suggested that Sh-lac was involved in the final step in the DHN melanin biosynthesis.

Reduced melanin inhibited the production of oxalic acid. Oxalic acid secretion by sclerotinia is deemed an essential determinant of its pathogenicity (Godoy et al. 1990; Noyes and Hancock 1981; Zhou and Boland 1999). Oxalic acid is a key pathogenicity factor in $S$. sclerotiorum and helps in the formation of sclerotia (Bolton et al. 2006; Cessna et al. 2000). Oxalic acid accumulates early during pathogenesis and increases in concentration as host colonization advances. Concomitant with the increase in oxalic acid is a decrease in extracellular host $\mathrm{pH}$ from 5 to 4, which enhances the activity of the fungus in the host tissues (Magro et al. 1984). S. shiraiana is also a sclerotia forming phytopathogen and the medium $\mathrm{pH}$ value reduced from 5.50 to 3.823 after 17 days inoculation in ME medium (Supplementary Table S2); therefore, we explored the possibility of oxalic acid production in medium. Our results showed WT produced $2.13 \mathrm{mM}$ of oxalic acid. However, oxalic acid was not detected in ilac-4, ilac-8, and CK. In addition, the oxalic acid content was significantly reduced in $S h-S C D$ silenced transformant and WT treated with tricyclazole. Since, oxalic acid secretion is consistent with melanin production in different transformants and WT, we infer that melanin is associated with oxalic acid production in S. shiraiana.

Conclusions. S. shiraiana is one of the main pathogens of mulberry popcorn disease. The sclerotia is formed by fractured hyphae of pathogen in diseased mulberry fruit. Sh-lac gene plays a key role in DHN catalysis to DHN melanin. Silenced Sh-lac gene resulted in inhibition of the production of melanin, vegetative growth, and oxalic acid. Our study indicated that the gene Sh-lac can be used as a target for mulberry popcorn disease control.

\section{ACKNOWLEDGMENTS}

This project was funded by the research grants from the National Hi-Tech Research and Development Program of China (2013AA100605-3), Natural Science Foundation of China (31572323), China Postdoctoral Science Foundation funded projects (2013M540694, 2014T70845, and 2016M592622), and the "111" Project (B12006).

\section{LITERATURE CITED}

Amselem, J., Cuomo, C. A., van Kan, J. A. L., Viaud, M., Benito, E. P., Couloux, A., Coutinho, P. M., de Vries, R. P., Dyer, P. S., Fillinger, S., Fournier, E., Gout, L., Hahn, M., Kohn, L., Lapalu, N., Plummer, K. M., Pradier, J.-M., Quévillon, E., Sharon, A., Simon, A., ten Have, A., Tudzynski, B., Tudzynski, P., Wincker, P., Andrew, M., Anthouard, V., Beever, R. E., Beffa, R., Benoit, I., Bouzid, O., Brault, B., Chen, Z., Choquer, M., Collémare, J., Cotton, P., Danchin, E. G., Da Silva, C., Gautier, A., Giraud, C., Giraud, T., Gonzalez, C., Grossetete, S., Güldener, U., Henrissat, B., Howlett, B. J., Kodira, C., Kretschmer, M., Lappartient, A., Leroch, M., Levis, C., Mauceli, E., Neuvéglise, C., Oeser, B., Pearson, M., Poulain, J., Poussereau, N., Quesneville, H., Rascle, C., Schumacher, J., Ségurens, B., Sexton, A., Silva, E., Sirven, C., Soanes, D. M., Talbot, N. J., Templeton, M., Yandava, C., Yarden, O., Zeng, Q., Rollins, J. A., Lebrun, M.-H., and Dickman, M. 2011. Genomic analysis of the necrotrophic fungal pathogens Sclerotinia sclerotiorum and Botrytis cinerea. PLoS Genet. 7: e 1002230.

Andersson, A., Jordan, D., Schneider, G., and Lindqvist, Y. 1996. Crystal structure of the ternary complex of 1,3,8-trihydroxynaphthalene reductase from Magnaporthe grisea with NADPH and an active-site inhibitor. Structure 4:1161-1170.

Baldrian, P. 2006. Fungal laccases-occurrence and properties. FEMS Microbiol. Rev. 30:215-242.

Bell, A. A., and Wheeler, M. H. 1986. Biosynthesis and functions of fungal melanins. Annu. Rev. Phytopathol. 24:411-451.

Blain, W. L. 1931. A list of diseases of economic plants in Alabama. Mycologia 23:300-304.

Bolton, M. D., Thomma, B. P. H. J., and Nelson, B. D. 2006. Sclerotinia sclerotiorum (Lib.) de Bary: Biology and molecular traits of a cosmopolitan pathogen. Mol. Plant Pathol. 7:1-16.

Breathnach, R., Benoist, C., O'Hare, K., Gannon, F., and Chambon, P. 1978. Ovalbumin gene: Evidence for a leader sequence in mRNA and DNA sequences at the exon-intron boundaries. Proc. Natl. Acad. Sci. USA 75: 4853-4857.

Butler, M. J., and Day, A. W. 1998. Fungal melanins: A review. Can. J. Microbiol. 44:1115-1136.

Butler, M. J., Day, A. W., Henson, J. M., and Money, N. P. 2001. Pathogenic properties of fungal melanins. Mycologia 93:1-8.

Butler, M. J., Gardiner, R. B., and Day, A. W. 2009. Melanin synthesis by Sclerotinia sclerotiorum. Mycologia 101:296-304.

Cessna, S. G., Sears, V. E., Dickman, M. B., and Low, P. S. 2000. Oxalic acid, a pathogenicity factor for Sclerotinia sclerotiorum, suppresses the oxidative burst of the host plant. Plant Cell 12:2191-2200.

Ebbole, D. J. 2007. Magnaporthe as a model for understanding host-pathogen interactions. Annu. Rev. Phytopathol. 45:437-456.

Edens, W. A., Goins, T. Q., Dooley, D., and Henson, J. M. 1999. Purification and characterization of a secreted laccase of Gaeumannomyces graminis var. tritici. Appl. Environ. Microbiol. 65:3071-3074.

Ellis, D. H., and Griffiths, D. A. 1974. The location and analysis of melanins in the cell walls of some soil fungi. Can. J. Microbiol. 20:1379-1386.

Ercisli, S., and Orhan, E. 2007. Chemical composition of white (Morus alba), red (Morus rubra) and black (Morus nigra) mulberry fruits. Food Chem. 103:1380-1384.

Godoy, G., Steadman, J. R., Dickman, M. B., and Dam, R. 1990. Use of mutants to demonstrate the role of oxalic acid in pathogenicity of Sclerotinia sclerotiorum on Phaseolus vulgaris. Physiol. Mol. Plant Pathol. 37: 179-191.

Gomez, B. L., and Nosanchuk, J. D. 2003. Melanin and fungi. Curr. Opin. Infect. Dis. 16:91-96.

Henson, J. M., Butler, M. J., and Day, A. W. 1999. The dark side of the mycelium: melanins of phytopathogenic fungi. Annu. Rev. Phytopathol. 37: 447-471.

Howard, R. J., and Valent, B. 1996. Breaking and entering: Host penetration by the fungal rice blast pathogen Magnaporthe grisea. Annu. Rev. Microbiol. 50:491-512.

Jacobson, E. S. 2000. Pathogenic roles for fungal melanins. Clin. Microbiol. Rev. 13:708-717.

Kihara, J., Moriwaki, A., Ito, M., Arase, S., and Honda, Y. 2004a. Expression of THR1, a 1,3,8-trihydroxynaphthalene reductase gene involved in melanin biosynthesis in the phytopathogenic fungus Bipolaris oryzae, is enhanced by near-ultraviolet radiation. Pigment Cell Res. 17:15-23.

Kihara, J., Moriwaki, A., Ueno, M., Tokunaga, T., Arase, S., and Honda, Y. 2004b. Cloning, functional analysis and expression of a scytalone dehydratase gene $(S C D 1)$ involved in melanin biosynthesis of the phytopathogenic fungus Bipolaris oryzae. Curr. Genet. 45:197-204.

Kim, S. B., Chang, B. Y., Jo, Y. H., Lee, S. H., Han, S.-B., Hwang, B. Y., Kim, S. Y., and Lee, M. K. 2013. Macrophage activating activity of pyrrole alkaloids from Morus alba fruits. J. Ethnopharmacol. 145:393-396. 
Kishi, K. 1998. Plant Disease in Japan. Zenkaku Noson Kyoiku Kyokai Co., Ltd., Tokyo, Japan.

Kohn, L. M., and Nagasawa, E. 1984. The genus Scleromitrula (Sclerotiniaceae), Episclerotium gen. nov. (Leotiacwae) and allied stipitate-capitate species with reduced ectal excipula. Trans. Mycol. Soc. Jpn. 25:23-38.

Langfelder, K., Streibel, M., Jahn, B., Haase, G., and Brakhage, A. A. 2003. Biosynthesis of fungal melanins and their importance for human pathogenic fungi. Fungal Genet. Biol. 38:143-158.

Lee, J.-K., Jung, H.-M., and Kim, S.-Y. 2003. 1,8-Dihydroxynaphthalene (DHN)-melanin biosynthesis inhibitors increase erythritol production in Torula corallina, and DHN-melanin inhibits erythrose reductase. Appl. Environ. Microbiol. 69:3427-3434.

Li, M., and Rollins, J. A. 2009. The development-specific protein (Ssp1) from Sclerotinia sclerotiorum is encoded by a novel gene expressed exclusively in sclerotium tissues. Mycologia 101:34-43.

Liao, D.-I., Basarab, G. S., Gatenby, A. A., Valent, B., and Jordan, D. B. 2001. Structures of trihydroxynaphthalene reductase-fungicide complexes: Implications for structure-based design and catalysis. Structure 9:19-27.

Lin, S. Y., Okuda, S., Ikeda, K., Okuno, T., and Takano, Y. 2012. LAC2 encoding a secreted laccase is involved in appressorial melanization and conidial pigmentation in Colletotrichum orbiculare. Mol. Plant-Microbe Interact. 25:1552-1561.

Liu, G. Y., and Nizet, V. 2009. Color me bad: Microbial pigments as virulence factors. Trends Microbiol. 17:406-413.

Magro, P., Marciano, P., and Di Lenna, P. 1984. Oxalic acid production and its role in pathogenesis of Sclerotinia sclerotiorum. FEMS Microbiol. Lett. 24: 9-12.

Mount, S. M. 1982. A catalogue of splice junction sequences. Nucleic Acids Res. 10:459-472.

Noyes, R. D., and Hancock, J. G. 1981. Role of oxalic acid in the Sclerotinia wilt of sunflower. Physiol. Plant Pathol. 18:123-132.

Özgen, M., Serçe, S., and Kaya, C. 2009. Phytochemical and antioxidant properties of anthocyanin-rich Morus nigra and Morus rubra fruits. Sci. Hortic. (Amsterdam) 119:275-279.

Perpetua, N. S., Kubo, Y., Yasuda, N., Takano, Y., and Furusawa, I. 1996. Cloning and characterization of a melanin biosynthetic THR1 reductase gene essential for appressorial penetration of Colletotrichum lagenarium. Mol. Plant-Microbe Interact. 9:323-329.

Sbaghi, M., Jeandet, P., Bessis, R., and Leroux, P. 1996. Degradation of stilbene-type phytoalexins in relation to the pathogenicity of Botrytis cinerea to grapevines. Plant Pathol. 45:139-144.

Siegler, E. A., and Jenkins, A. E. 1923. Sclerotinia carunculouloides, the cause of a carious disease of the mulberry (Morus alba). J. Agric. Res. 23: 833-836.

Tanaka, C., Kubo, Y., and Tsuda, M. 1991. Genetic analysis and characterization of Cochliobolus heterostrophus colour mutants. Mycol. Res. 95: 49-56.

Tanaka, C., Tajima, S., Furusawa, I., and Tsuda, M. 1992. The Pgr1 mutant of Cochliobolus heterostrophus lacks a p-diphenol oxidase involved in naphthalenediol melanin synthesis. Mycol. Res. 96:959-964.

Tetsch, L., Bend, J., Janßen, M., and Hölker, U. 2005. Evidence for functional laccases in the acidophilic ascomycete Hortaea acidophila and isolation of laccase-specific gene fragments. FEMS Microbiol. Lett. 245:161-168.

Thomma, B. P. H. J. 2003. Alternaria spp.: From general saprophyte to specific parasite. Mol. Plant Pathol. 4:225-236.
Thompson, J. E., Fahnestock, S., Farrall, L., Liao, D.-I., Valent, B., and Jordan, D. B. 2000. The second naphthol reductase of fungal melanin biosynthesis in Magnaporthe grisea: Tetrahydroxynaphthalene reductase. J. Biol. Chem. 275:34867-34872.

Thywissen, A., Heinekamp, T., Dahse, H. M., Schmaler-Ripcke, J., Nietzsche, S., Zipfel, P. F., and Brakhage, A. A. 2011. Conidial dihydroxynaphthalene melanin of the human pathogenic fungus Aspergillus fumigatus interferes with the host endocytosis pathway. Front. Microbiol. 2:96.

Upadhyay, S., Torres, G., and Lin, X. 2013. Laccases involved in 1,8dihydroxynaphthalene melanin biosynthesis in Aspergillus fumigatus are regulated by developmental factors and copper homeostasis. Eukaryot. Cell 12:1641-1652.

Vidal-Cros, A., Viviani, F., Labesse, G., Boccara, M., and Gaudry, M. 1994. Polyhydroxynaphthalene reductase involved in melanin biosynthesis in Magnaporthe grisea. Eur. J. Biochem. 219:985-992.

Watt, A. A. R., Bothma, J. P., and Meredith, P. 2009. The supramolecular structure of melanin. Soft Matter 5:3754-3760.

Wheeler, M. H., and Stipanovic, R. D. 1979. Melanin biosynthesis in Thielaviopsis basicola. Exp. Mycol. 3:340-350.

Whetzel, H. H., and Wolf, F. A. 1945. The cup fungus, Ciboria-Carunculoides, pathogenic on mulberry fruits. Mycologia 37:476-491.

White, T. J., Bruns, T., Lee, S., and Taylor, J. W. 1990. Amplification and direct sequencing of fungal ribosomal RNA genes for phylogenetics. Pages 315-322 in: PCR Protocols: A Guide to Methods and Applications. Academic Press, San Diego, CA.

Williamson, P. R., Wakamatsu, K., and Ito, S. 1998. Melanin biosynthesis in Cryptococcus neoformans. J. Bacteriol. 180:1570-1572.

Wu, Y., Shan, L., Yang, S., and Ma, A. 2008. Identification and antioxidant activity of melanin isolated from Hypoxylon archeri, a companion fungus of Tremella fuciformis. J. Basic Microbiol. 48:217-221.

Yelton, M. M., Hamer, J. E., and Timberlake, W. E. 1984. Transformation of Aspergillus nidulans by using a trpC plasmid. Proc. Natl. Acad. Sci. 81: 1470-1474.

Yu, Y., Jiang, D., Xie, J., Cheng, J., Li, G., Yi, X., and Fu, Y. 2012. Ss-S12, a novel cell wall protein with PAN modules, is essential for sclerotial development and cellular integrity of Sclerotinia sclerotiorum. PLoS One 7: e34962.

Zhang, G., Ma, L., Beuchat, L. R., Erickson, M. C., Phelan, V. H., and Doyle, M. P. 2009. Evaluation of treatments for elimination of foodborne pathogens on the surface of leaves and roots of lettuce (Lactuca sativa L.). J. Food Prot. 72:228-234.

Zhou, T., and Boland, G. J. 1999. Mycelial growth and production of oxalic acid by virulent and hypovirulent isolates of Sclerotinia sclerotiorum. Can. J. Plant Pathol. 21:93-99.

\section{AUTHOR-RECOMMENDED INTERNET RESOURCES}

SignalP 4.1 Server: http://www.cbs.dtu.dk/services/SignalP/

MultiLoc: http://abi.inf.uni-tuebingen.de/Services/MultiLoc/

Search for Conserved Domains:

http://www.ncbi.nlm.nih.gov/Structure/cdd/wrpsb.cgi

Broad Institute's Fungal Genome Initiative (FGI):

http://www.broadinstitute.org/annotation/fgi/

GenBank: http://www.ncbi.nlm.nih.gov/genbank/ 